\title{
Das Spät- und Postglazial im Berninapaßgebiet
}

\section{Einleitung}

Der Berninapaß verbindet nordöstlich der Berninagruppe in den südrätischen Alpen das Engadin mit dem Veltlin. In der weiteren Umgebung der Paßhöhe finden wir typische Elemente der Paßlandschaft: breite, rundgeschliffene Übergänge und mehrere Seen. Der künstlich vergrößerte Lago Bianco (Weißer See) sammelt trübes Gletscherwasser. Der benachbarte Lej Nair (Schwarzer See) sowie die übrigen kleineren Seen werden von Quellwasser gespiesen. Mit ihrem moorigen Grund sehen sie dunkel aus. Das Längsprofil des Passes ist extrem asymmetrisch: Vom Oberengadin (1750 $\mathrm{m}$ ü. M.) steigt das Tal - abgesehen von der Stufe bei der Mündung des Morteratschtales gleichmäßig bis zur Paßhöhe (2328 $\mathrm{m}$ ü. M.) und fällt dann steil gegen das auf ca. $1000 \mathrm{~m}$ ü. M. gelegene obere Puschlav.

Der Berninapaß befindet sich im wesentlichen im Bereich der unterostalpinen Berninadecke im weiteren Sinn. Sie weist hier zwei Digitationen auf. Die untere, die Berninadecke im engeren Sinn, besteht im Paßgebiet vor allem aus Alkaligraniten. Die obere, die Strettadecke, ist überwiegend aus Orthogneisen aufgebaut. Getrennt werden die beiden Digitationen durch Sedimente der Trias und des Lias (hauptsächlich Dolomit). Die untere Sedimentserie (Alv-Zug) ist die Bedeckung der Berninadecke im engeren Sinn, während die obere (Gessi-Zug) auf dem Kristallin der Strettadecke liegt. Im nördlichen Bereich wird die Berninadecke durch den Alv-Zug von der mittelbzw. oberostalpinen Languarddecke getrennt.

Der Berninapaß ist eine markante Klimascheide. Das Oberengadin zeigt ausgeprägte kontinentale Züge, wie sie inneralpinen Tälern im Bereich großer Massenerhebung eigen sind. Das Puschlav weist insubrische Züge auf, erhält aber nicht so viel Niederschlag wie typische nach Süden offene Alpentäler.

Die Waldgrenze befindet sich im Oberengadin auf $2200 \mathrm{~m}$ ü. M.; sie wird durch Lärchen und Arven gebildet. Im Puschlav besteht die Waldgrenze nur aus Lärchen; sie ist auf $2100 \mathrm{~m}$ ü. M. anzusetzen. Im eigentlichen Berninapaßgebiet findet man die zu erwartende Depression der Höhenstufen.

Fridolin Beeler, Dr. phil. II, St. Jakobstraße 62, 6330 Cham.

\section{Gletschergeschichtliche Untersuchungen}

\subsection{Vorbemerkung}

Hauptgegenstand der Untersuchungen war die Gletschergeschichte. Besondere Aufmerksamkeit zogen der Palü- und der Cambrena-, ferner der MorteratschGletscher auf sich.

\subsection{Die Eistransfluenz über den Berninapaß}

Individuelle Spuren hinterließen die Gletscher erst nach dem Zerfall des Eisstromnetzes. Die hochliegende Schliffgrenze (mehr als $3000 \mathrm{~m}$ ü. M.) um Pontresina weist auf eine Eiskulmination, von der die Eisströme durch alle Täler abflossen, so auch über den Bernina$\mathrm{pa} ß$ in den breiten Talschluß des Puschlavs und durch das Heutal ins Livignotal. Entsprechendes Erratikum ist vorhanden, prachtvolle nord-südgerichtete Gletscherschliffe blieben erhalten. Der Abschluß der Eistransfluenz liegt im Dunkeln, hat sich aber auch hier - in der Umgebung einer Eiskulmination - wahrscheinlich nicht nach dem Bölling vollzogen.

\subsection{Die Moränenkartierung}

Wichtigste gletschergeschichtliche Hinweise sind Moränenwälle. KINZL (1949) hat klargestellt, daß nur Gletschervorstöße zu Moränenwällen führen. Eine kritische Kartierung dieser Formen ist daher unumgänglich. Sie führte im Arbeitsgebiet zur Gruppierung von vier Ständen. Die Zuordnung geschah - wo möglich - durch Schneegrenzberechnungen, ferner durch Schneegrenzabschätzungen, nach der morphologischen Ausbildung und Größenordnung und durch ${ }^{14} \mathrm{C}$-Daten. Es sei vorweggenommen, daß es sich bei den mit Lokalnamen bezeichneten Ständen «Val-daFain» und «Bernina-Suot» einerseits, «Languard» sowie beim «Cavaglia-Stadium» andererseits um spätglaziale Wälle handelt, welche Schneegrenz-Depressionen von ca. $300 \mathrm{~m}$ bzw. 160-240 m gegenüber dem Hochstand um 1850 aufweisen. Entsprechende Moränen wurden am Palügletscher spätestens im mittleren Präboreal abgelagert. Eine weitere Gruppe (Morteratsch-Stand) ergab Schneegrenz-Depressionen von 50 bis $120 \mathrm{~m}$ gegen 1850 . Die Schneegrenz-Depressionen der genannten Gruppen stimmten mit den Ergebnissen von HANTKE (1978) überein. Bei der letzten Gruppe 


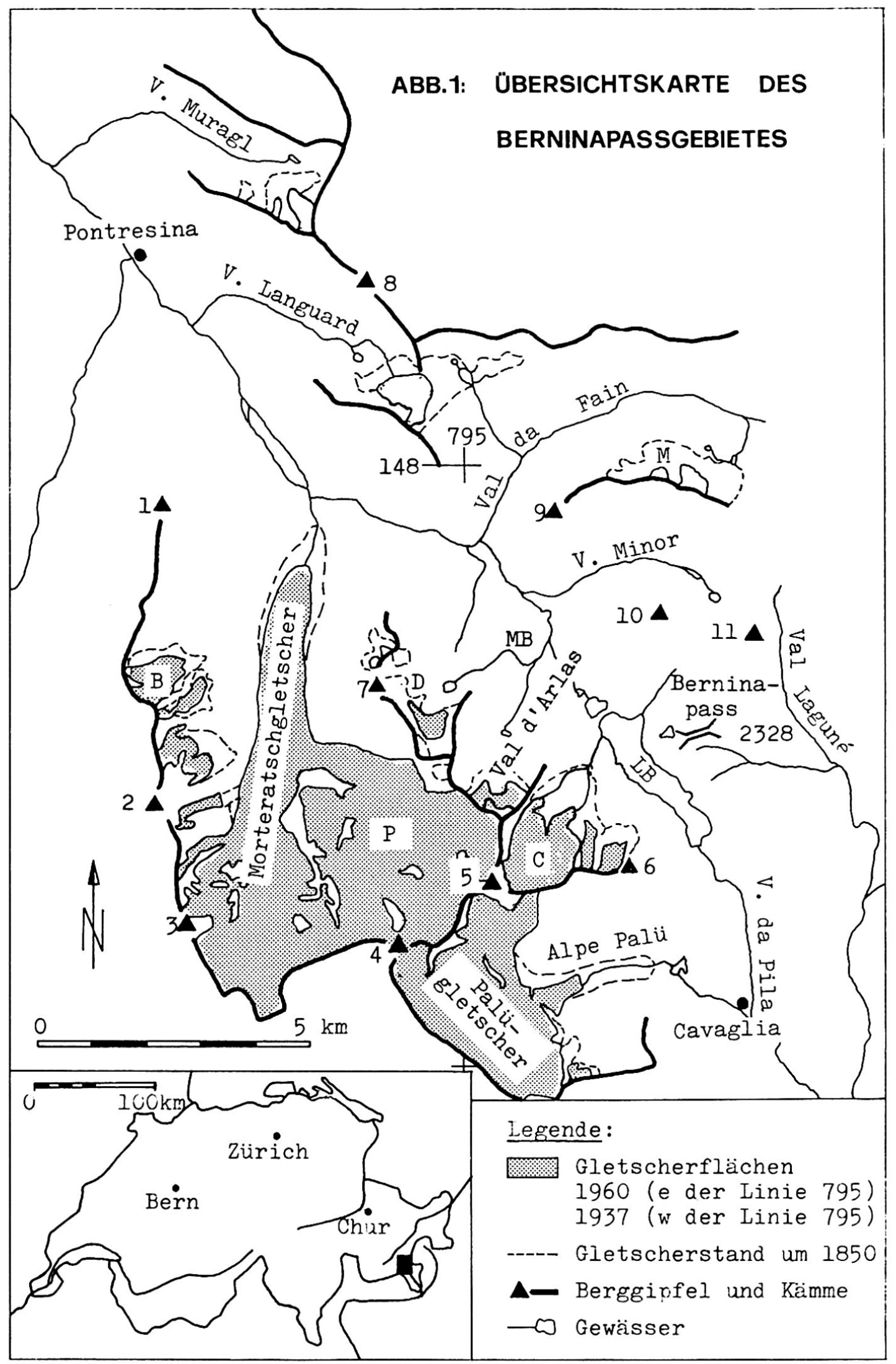

B Bovalgletscher

C Cambrenagletscher

D Diavolezza

LB Lago Bianco

$M$ Minorgletscher

MB Moor Buottels

$\mathrm{P}$ Persgletscher
1 Piz Chalchagn (3154 m ü. M.)

2 Piz Morteratsch (3751 m ü. M.)

3 Piz Bernina (4049 m ü. M.)

4 Piz Palü (3905 m ü. M.)

5 Piz Cambrena (3604 m ü. M.)

6 Sassal Mason (3032 m ü. M.)

7 Munt Pers (3207 m ü. M.)
8 Piz Languard (3262 $\mathrm{m}$ ü. M.)

9 Piz Alv (2975 m ü. M.)

10 Piz Lagalb (2959 m ü. M.)

11 Geß (2412 mü. M.) 
Gletscherhochstände und -vorstöße im Berninapaßgebiet

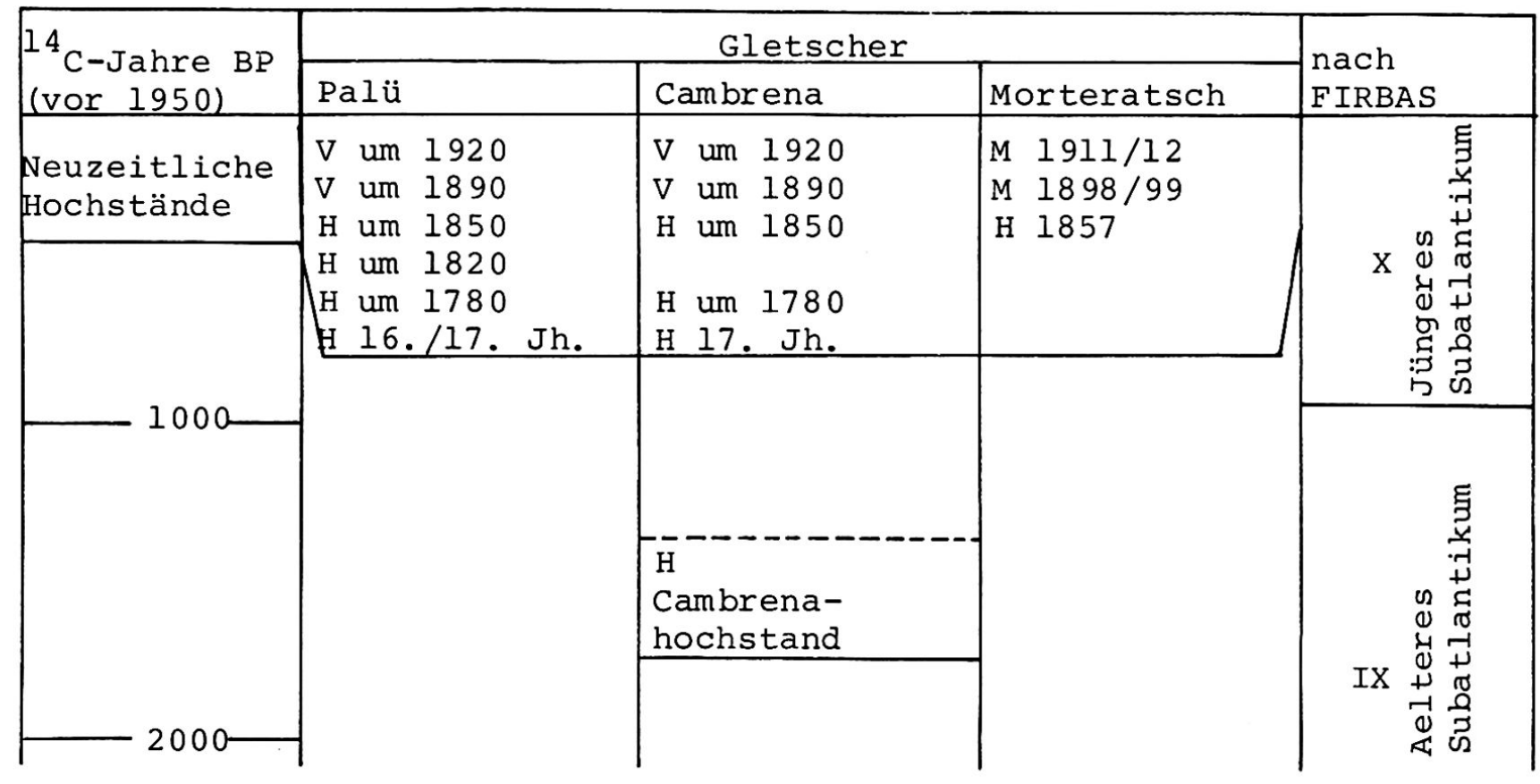
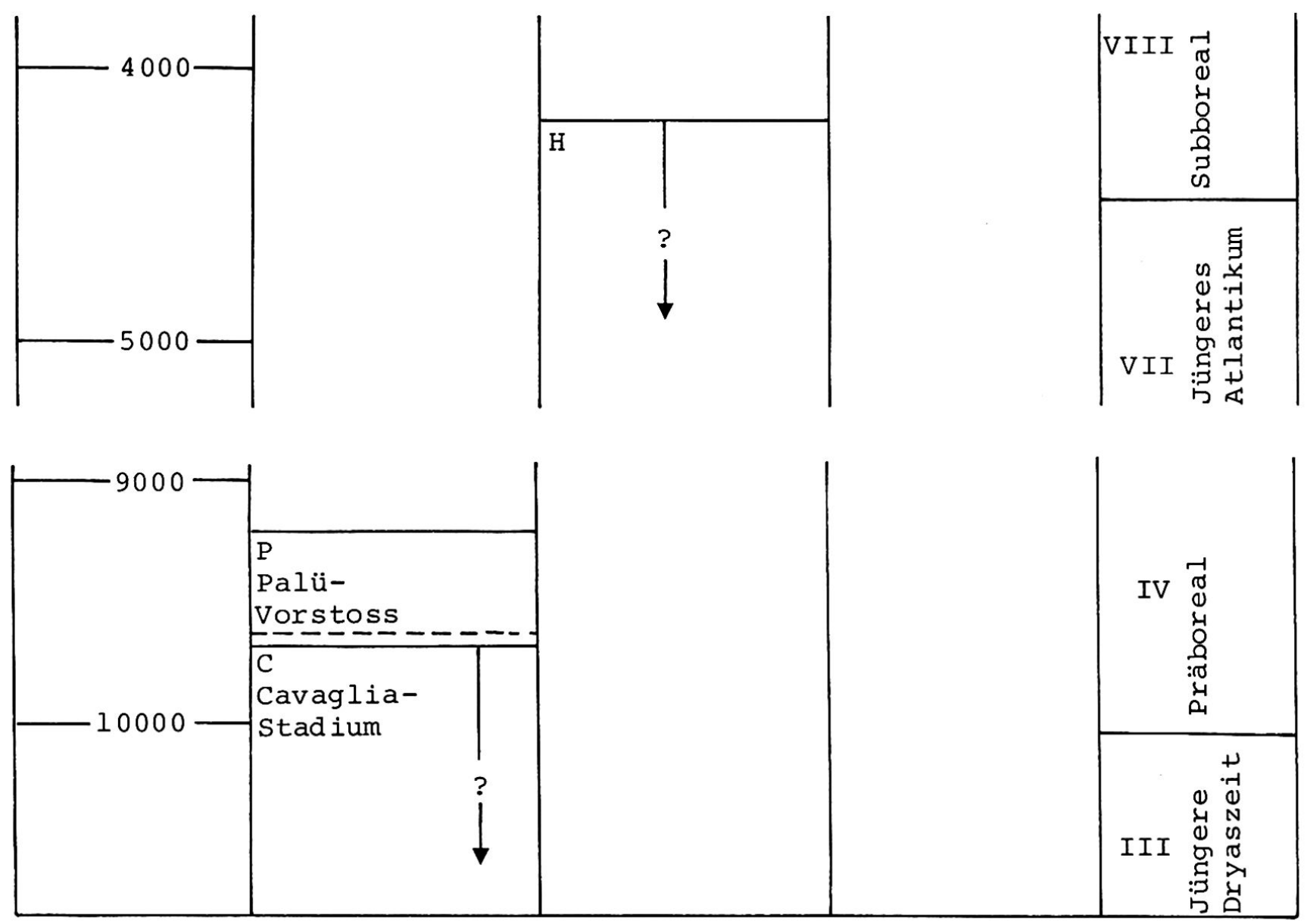

$\mathrm{H}$ : durch Endmoränen dokumentierter Gletscherhochstand. Größenordnung wie um 1850.

V: durch Endmoränen dokumentierter Gletschervorstoß. Größenordnung kleiner als um 1850.

M: unbedeutende durch Gletschermessungen festgestellte Vorstöße, welche nur undeutliche Moränensysteme im Innern des Gletschervorfeldes hinterlassen haben.

P: Gletscherhochstand etwas größer als um 1850. Endmoränen nicht erhalten. Pollenanalytisch nachgewiesen von H.ZOLLER. 
handelt es sich um postglaziale Moränenwälle, welche sich auf den Gletschervorfeldern im Sinne von KINZL (1949) oder in deren nahen Umgebung befinden (mittleres Präboreal und jünger).

\subsection{Das Spätglazial}

2.4.1. Bernina-Suot- und Val-da-Fain-Stand: Deutliche Endmoränen von Vorstößen der Lokalgletscher über die Größenordnung der neuzeitlichen Hochstände hinaus sind auf der Moränenkarte dem LanguardStand und dem Cavaglia-Stadium zugeordnet. Auf der Nordseite des Passes konnten außerhalb dieses Standes Hinweise auf einen größeren Gletscherstand gefunden werden: höher gelegene Seitenmoränen, Moränenablagerungen am Eingang ins Heutal, welche auf einen Gletscher weisen, der talaufwärts ins Heutal eindrang. Als klarsten Hinweis auf die Ausdehnung des entsprechenden Gletschers bot sich die Verbreitung des Alv-Gessi-Erratikums an. Der Alv-Gessi-Zug quert das Berninapaßtal im Bereich der Endmoränen des Languard-Standes. Jeder lokale Gletschervorsto $B$ mußte diese Sediment-Erratiker mit sich führen. Überraschenderweise fehlt das Erratikum unterhalb der Punt da Bernina bei den Berninahäusern, mehr als $1 \mathrm{~km}$ oberhalb der Einmündung des Morteratschtales. Dieses Ergebnis konnte als Hinweis auf die Ausdehnung eines Lokalgletschervorstoßes gewertet werden, der mit dem romanischen Namen der Lokalität Berninahäuser «Bernina-Suot» bezeichnet wurde. Schneegrenz-Abschätzungen im Paßgebiet - Berechnungen sind wegen der unbekannten Gletscherausdehnung nach Süden nicht möglich - im weiteren Vergleiche mit einem rekonstruierten Gletscherstand im Heutal (Val-da-Fain) ergaben, daß es sich um denselben Gletscherstand handeln könnte: Schneegrenzdepressionen gegen $1850 \mathrm{ca} .300 \mathrm{~m}$. Über die zeitlichen Verhältnisse des Bernina-Suot-Standes ist noch nichts gesichert. Falls im Moor Buottels das Alleröd nachgewiesen werden könnte - was aufgrund der Pollenanalyse einer Einzelprobe nach zoLLER (mdl. Mitt.) nicht ausgeschlossen werden kann, müßte der Stand in die ältere Dryaszeit gesetzt werden. Die Stelle des Moores lag zur Zeit des Hochstandes unter ca. $80 \mathrm{~m}$ mächtigem Eis.

Ein größerer Gletscherstand als das Cavaglia-Stadium läßt sich auch auf der Südseite nachweisen: Damals muß in Cavaglia ein Gletscher aus dem Paßgebiet, der durch das Val da Pila floß, noch mit dem Palügletscher zusammengekommen sein. Die scharfkantigen Moränenwälle am Ausgang des Val da Pila sind jedoch eindeutige Seitenmoränen des Palügletschers.

2.4.2. Das Cavaglia-Stadium und der Languard-Stand: Die besagten Wälle gehören nach den Rekonstruktionen zu einem Gletscherende in Cavaglia, das durch eine klare Endmoräne belegt ist (Cavaglia-Stadium, vgl. Abb. 2). Dazu dürften deutliche Seitenmoränen auf der Alpe Palü (vgl. Abb.3) gehören, für welche zwei Mindestalter vorliegen. $140 \mathrm{~m}$ nordöstlich über den neuzeitlichen Endmoränen staut einer dieser Seitenmoränenwälle ein 3,5 m tiefes Moor. Die Basisprobe ergab das ${ }^{14} \mathrm{C}$-Alter $9635 \pm 160 \mathrm{Jahre} \mathrm{BP}$ (UZ-46). Im weiteren liegt nur $400 \mathrm{~m}$ östlich der neuzeitlichen Endmoränen des Palügletschers ein Moor, dessen organische Basis mit $9460 \pm 140{ }^{14} \mathrm{C}$-Jahre BP (B-2635) datiert wurde. Aufgrund dieser Daten kann das Cavaglia-Stadium spätestens in das mittlere Präboreal gestellt werden. Berücksichtigt man die Zeit des Abschmelzens von Cavaglia (1700 m ü. M.) bis auf die Alpe Palü (1950 m ü. M.), so kann für die Zeit der Ablagerung der Moränen die jüngere Dryaszeit nicht ausgeschlossen werden. Damit liegt die Korrelation des Cavaglia- mit dem Egesen-Stadium nahe (vgl. KERSCHNER, 1978).

Im Moor Buottels befindet sich unter dem 2,25 m mächtigen organischen Sediment eine 1,25 m dicke Tonschicht. Die Basis der organischen Sedimentation ergab das ${ }^{14} \mathrm{C}$-Alter $9520 \pm 130 \mathrm{Jahre}$ BP (UZ-287). Damit steht das Mindestalter der äußeren LanguardStände des Arlasgletschers fest; sie wurden spätestens im mittleren Präboreal abgelagert.

\subsection{Das Postglazial}

2.5.1. Der Palü-Vorstoß: Das Moor auf der Alpe Palü, aus dem das Datum $9460 \pm 140$ stammt, ist von Herrn Prof. Dr. H.ZOLLER pollenanalytisch bearbeitet worden (ZOLLER, in Vorbereitung). Aus der Situation des Moores, seiner Stratigraphie und dem Erhaltungszustand der Pollen kann angenommen werden, daß der Palügletscher unmittelbar vor dem ${ }^{14} \mathrm{C}$-Datum bis auf das Niveau des Moores reichte, bald Schmelzwasser in das Moor lieferte, bald etwas dünner war, daß sich im Moor Gyttja-Horizonte bilden konnten. Das Moorprofil zeigt eine Wechsellagerung von Gyttja und Silt, in der sich Anzeichen einzelner Pioniergehölze befinden. Mit dem Datum beginnt der Diagramm-Abschnitt, der auf postglaziale Verhältnisse auf der Alpe Palü weist: Der Palügletscher ist auf neuzeitliche GröBenordnung abgeschmolzen.

Bedeutungsvoll sind die untersten, rein anorganischen Horizonte des Profils: Nachdem sich eine lockere Pioniervegetation abzeichnet, wird die Pollenfrequenz besonders gering und der Erhaltungszustand der Pollen sehr schlecht, was zoller (schriftl. Mitt.) als Anzeichen für einen Gletschervorstoß (Palü-Vorstoß) deutet, welcher sich kurz vor $9460 \pm 140$ Jahren BP abgespielt hat. Entsprechende - wenn auch unklare Moränenablagerungen sind vorhanden. Der Gletschervorstoß könnte dem Schlaten-Vorstoß (PATZELT, 1977) entsprechen. Wesentlich ist das Ergebnis, daß seit dem ${ }^{14} \mathrm{C}$-Datum der Palügletscher nie mehr sehr viel größer war als während der neuzeitlichen Hochstände. 


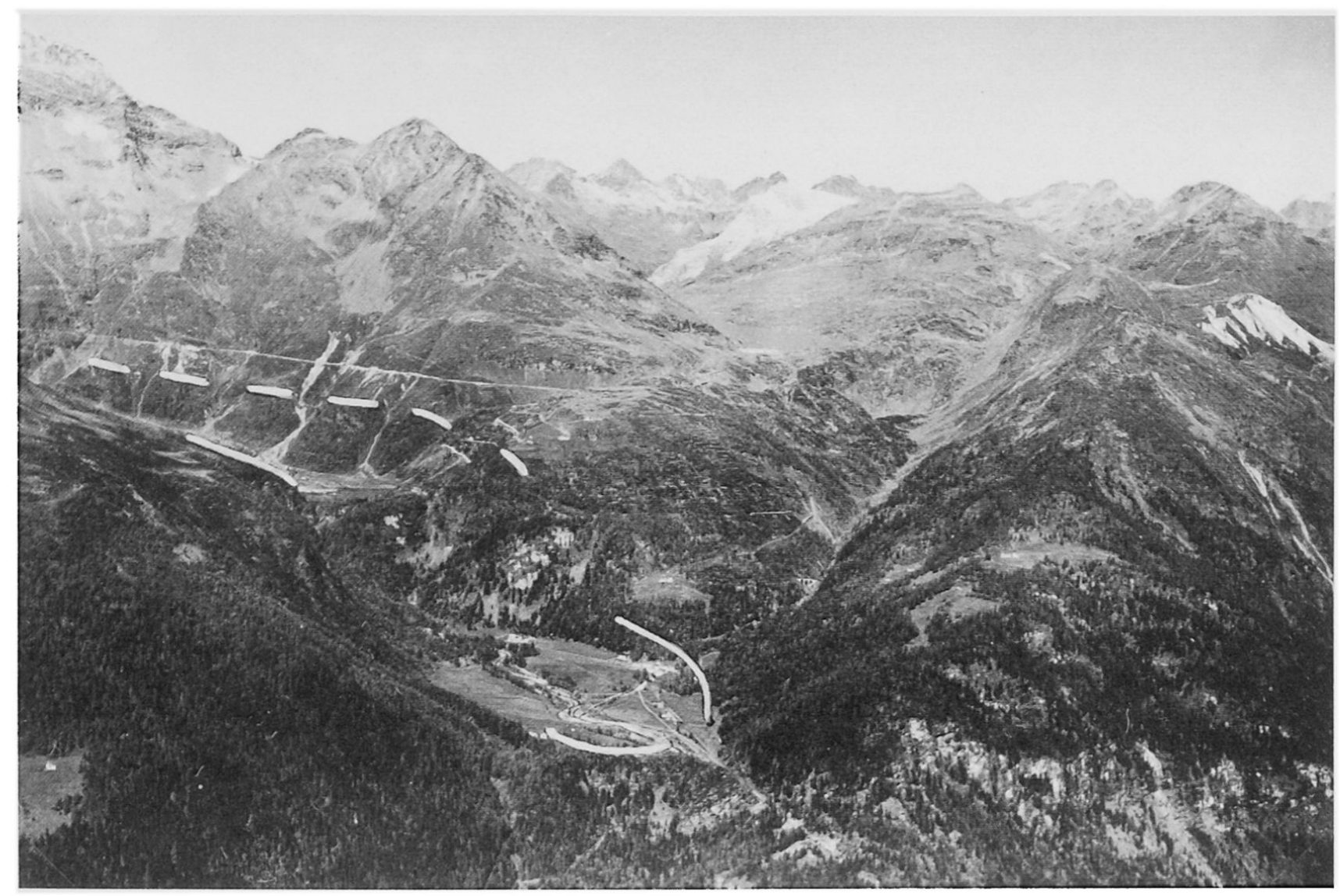

Abb. 2: Blick von Süden auf den Berninapaß. Rechter Bildrand: Sedimentkuppe der Geß; über der Bildmitte: Sedimentberg Piz Alv; Pyramide links davon: Piz Languard. Über der Alpe Palü (linke Bildseite): Sassal Mason. Mündung des Val da Pila in den Talkessel von Cavaglia. Durch weiße Striche sind die Moränen des Cavaglia-Stadiums des Palügletschers verdeutlicht. Photo Swissair.

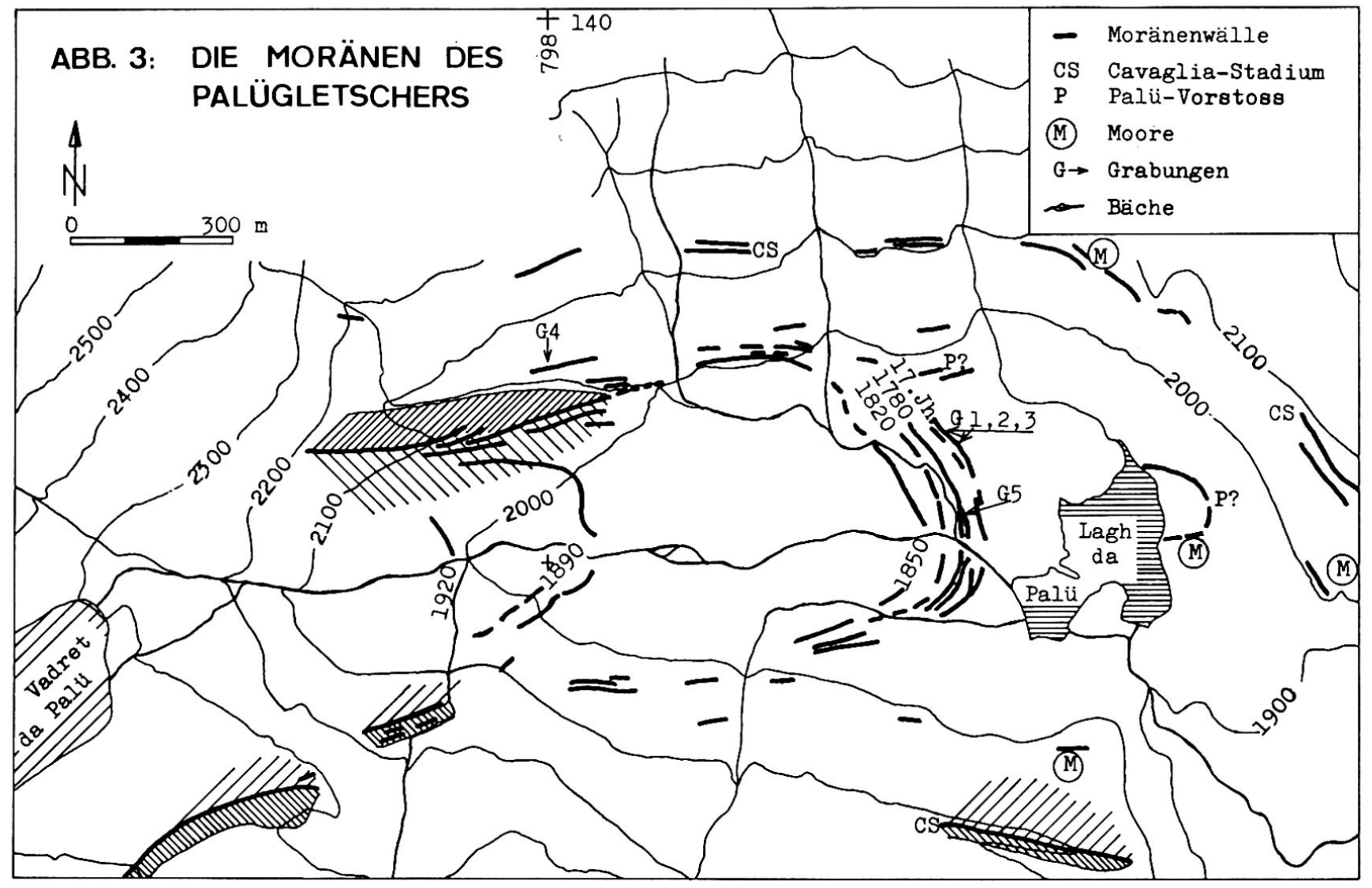


2.5.2. Der Hochstand A des Cambrenagletscher (vgl. Abb. 4): Das älteste ${ }^{14} \mathrm{C}$-Datum vom Gletschervorfeld des Cambrenagletschers ergab der Basistorf eines kleinen Moores, unmittelbar außerhalb der begrenzenden Moränenwälle. Das Moor wird durch die Moräne A gestaut. Daher beträgt ihr Mindestalter $4340 \pm 110$ Jahre BP (B-2532). Sie wurde demnach spätestens am Ende der postglazialen Wärmezeit gebildet. Das Gletschervorfeld ist äußerst scharf von der Umgebung abgegrenzt, wo keine Moränenwälle zu beobachten sind (vgl. Abb. 5). Auf der Nordseite befindet sich außerhalb der Moränenwälle ein Graben, der offenbar zur Zeit von Gletscherhochständen Schmelzwasser ableitete. Die Grabenböschung weist bis $25 \mathrm{~cm}$ mächtige verbraunte Horizonte auf, welche die Anlage des Grabens als vorneuzeitlich wahrscheinlich machen. Der Untergrund der alpinen Matten außerhalb des Gletschervorfeldes enthält über der Grundmoräne eine bis $1,1 \mathrm{~m}$ mächtige fluviale Verschüttungsserie, welche unter der rezenten Bodenbildung zwei bis $50 \mathrm{~cm}$ mächtige verbraunte Horizonte, getrennt durch graue Schuttschichten, zeigt. Da die Moräne A die älteste nachgewiesene Moräne im Bereich des Gletschervorfeldes ist, deren Alter zwar nicht feststeht, aber mindestens spätwärmezeitlich ist, kann nicht ausgeschlossen werden, daß das ganze Postglazial des Cambrenagletschers auf das Gletschervorfeld beschränkt ist.

2.5.3. Der Cambrena-Hochstand (vgl. Abb. 4): Im erwähnten Moor beim Vorfeld des Cambrenagletschers trennt eine tonig-sandige Einschwemmung die oberen, humos-torfigen Horizonte vom dichten Torf, der den Grund ausfüllt. Direkt von der Einschwemmung eingedeckt und im grünen Zustand konserviert wurde das Moos an der Oberfläche des Torfes, welches das ${ }^{14} \mathrm{C}$ Datum $1490 \pm 100$ BP (B-2531) besitzt. Die Einschwemmung läßt sich bis in die Moräne $C$ verfolgen. Damit ist der Hochstand, welcher den Moränenwall aufschüttete (Cambrena-Hochstand), datiert. Die Basis des humos-torfigen Materials über der Einschwemmung ergab das ${ }^{14}$ C-Datum $1570 \pm 55$ Jahre BP (Hv-6129). Der Unterschied der beiden Daten liegt innerhalb der Fehlergrenze. Die Inversion mag im Unterschied des Materials begründet sein; es kann nicht ausgeschlossen werden, daß Humus in der Umgebung aberodiert und eingeschwemmt worden ist. Auch die tonig-sandige Einschwemmung enthält feine braune Schichten, die auf gleiche Weise erklärt werden können. Gestützt wird das Alter des Cambrena-Hochstandes durch das Datum eines fossilen $A_{h}$-Restes, der durch die Moräne $C$ verschüttet worden ist und $1735 \pm 80$ ${ }^{14} \mathrm{C}$-Jahre BP (UZ-47) ergab. Es handelt sich um das Maximaldatum der Verschüttung durch die CambrenaHochstandsmoräne.

Das ${ }^{14} \mathrm{C}$-Datum $440 \pm 50$ Jahre BP (UZ-35) schloß aus, $\mathrm{da} ß$ die neuzeitlichen Hochstände Material für die tonigsandige Einschwemmung lieferten.
Abb. 4: Profil durch das Cambrena-Moor und die Moränen am Rand des Gletschervorfeldes.

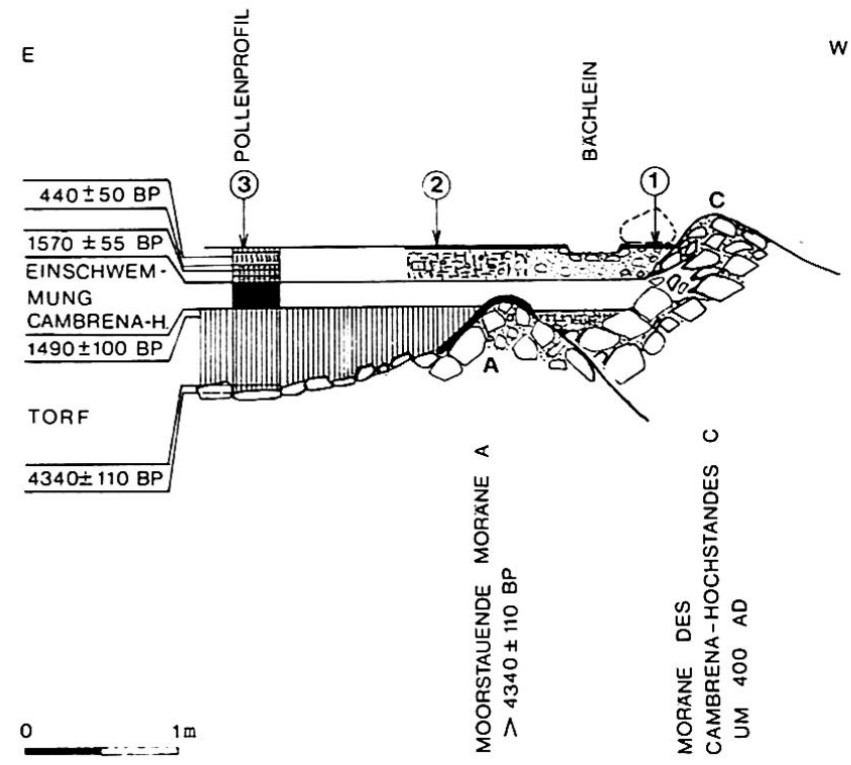

Im Pollenprofil entsprechen die Signaturen dem Pollendiagramm Cambrena. Im übrigen gilt folgende Legende:

His humos-torfiges Material

Einschwemmung Cambrena- $\mathrm{H}$. =

Ton und Sand, der zur Zeit des Gletscherhochstandes der Cambrena-Schwankung ins Moor floß.

torfige Bodenbildung

(1)-(3) Grabungen

2.5.4. Mittelalterliche Gletscherhochstände: Für die Zeit zwischen dem Cambrena-Hochstand und der Neuzeit gibt es im Berninapaßgebiet nur schwache Hinweise: Am Cambrenagletscher ergab die Datierung eines Bodens, der spätestens in der Neuzeit durch einen Moränenwall eingedeckt wurde, $1215 \pm 45{ }^{14} \mathrm{C}$ Jahre BP (Hv-6130). Am Palügletscher war zusammengeschwemmtes humoses Material aus einem an der Oberfläche neuzeitlichen Moränenwall, der aber möglicherweise Reste älterer Seitenmoränen enthält, $698 \pm 59{ }^{14} \mathrm{C}$-Jahre (Eidgenössische Forschungsanstalt für landwirtschaftlichen Pflanzenbau, Zürich-Reckenholz) alt. Beide Daten sind Maximalalter für die Verschüttung durch das darüberliegende Moränenmaterial. Somit können mittelalterliche Gletscherhochstände im Berninapaßgebiet nicht ausgeschlossen werden.

2.5.5. Neuzeit (vgl. Abb. 5 und 6): Vor dem Beginn der neuzeitlichen Vorstöße dürften die Gletscher des Berninapaßgebietes während längerer Zeit kleiner als beim größten neuzeitlichen Hochstand gewesen sein. Sowohl am Cambrena- als auch am Palülgletscher wurden durch den ersten neuzeitlichen Vorstoß Bodenbildungen zusammengeschoben, für deren Bildung 


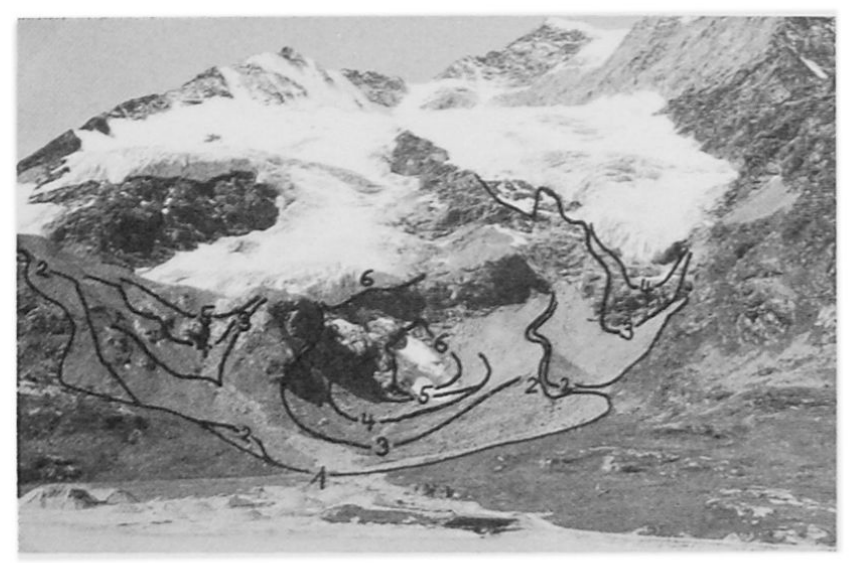

Abb. 5: Cambrenagletscher mit Piz Cambrena (rechts) und Piz Caral (links). Aufnahme: Sommer 1974. Die schwarzen Striche veranschaulichen die neuzeitliche Entwicklung des Gletschers, besonders seit 1850. Bedeutung der Zahlen: 1: um 1780; 2: um 1850; 3: 1880-1900; 4: 1907; 5: um 1930; 6: 1955 .

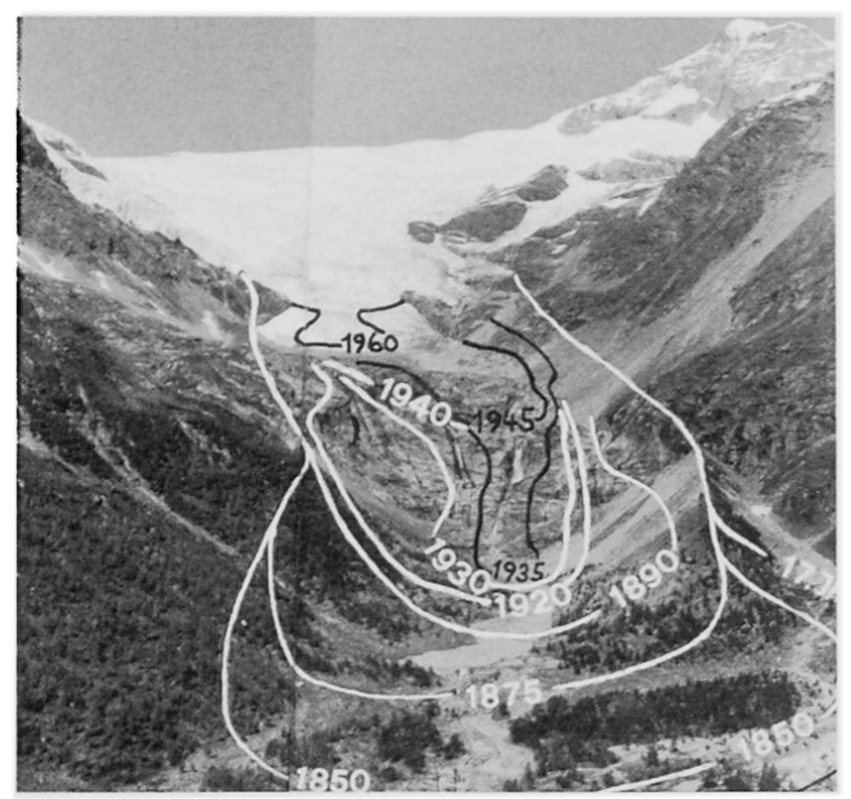

Abb.6: Der Palügletscher mit Piz Palü (rechts oben). Aufnahme: um 1974. Die schwarzen Striche zeigen die neuzeitliche Entwicklung des Gletschers, besonders seit 1850. Vor alle Jahrzahlen muß «um» gesetzt werden.

einige Jahrhunderte veranschlagt werden müssen: Am Cambrenagletscher besteht die äußerste neuzeitliche Moräne neben Blöcken fast nur aus Humus und Teilen des B-Horizontes der alten Landoberfläche. Am Palügletscher wurden die Sedimente der Alpe Palü faltenartig zusammengestaucht. HAEBERLI (1979, S. 46) schreibt, daß derartige Zusammenschübe während des Winters erfolgen, wenn der Boden an der Oberfläche gefroren ist. Diese Erklärung läßt verstehen, warum derart saubere Faltenstrukturen resultieren.

Ein fossiler Boden aus diesen Faltungen ergab das ${ }^{14} \mathrm{C}$-Datum $360 \pm 80$ Jahre BP (Hv-6131) und stellt somit ein Maximalalter für den Zusammenschub dar. Auf dem Moränenwall, vor dem die erwähnten
Stauchungswülste liegen, befindet sich ein Alpgebäude mit der Jahrzahl 1779. Der Alpstall wurde wohl dort errichtet, wo seit Menschengedenken keinEis mehr lag. Mit großer Wahrscheinlichkeit wurde daher der Wall im 16. oder 17. Jahrhundert gebildet.

Die Flechten auf den Moränenwällen des Hochstandes um 1850 dienten als Basis für die lichenometrische Datierung der neuzeitlichen Moränen. Die Ausdehnung der Gletscher kurz vor dem Hochstand kann dem Meßtischblatt für die Dufourkarte entnommen werden, das J. R. STENGEL 1849 bearbeitet hat. Deutlich werden darauf Schotterflächen und alpine Matten auseinandergehalten. Die Einbuchtung des Cambrenagletschers auf der Ostseite (vgl. Abb.5), welche sich im entsprechenden Moränenwall widerspiegelt, ist auf dem Meßtischblatt gut zu erkennen. Der Hochstand um 1850 hat am Palü-, Cambrena- und Morteratschgletscher zum innersten Wallsystem am Rand des Gletschervorfeldes geführt, vorausgesetzt, daß der Vorstoß überhaupt einen Stirnmoränenwall erzeugte. (Der andere Fall ist eine selten beobachtete Ausnahme!) Nach der lichenometrischen Bestimmung gibt es im Vorfeld des Palügletschers Wälle aus den Jahren um 1820 und um 1780. (Das äußerste Wallsystem, auf dem das Alpgebäude mit der Jahrzahl 1779 steht, wurde wegen der durch die Alpwirtschaft veränderten ökologischen Verhältnisse für Flechtenmessungen nicht beigezogen.) Am Rand des Cambrenagletschervorfeldes stammt ein Wallsystem aus der Zeit um 1780 und eines aus der ersten Hälfte des 17. Jahrhunderts. Die Moränenwälle im Innern der Gletschervorfelder konnten mit Hilfe von Gletschermessungen, Kartenaufnahmen, der Baugeschichte der Kraftwerke Brusio und der Berninabahn, zusammen mit entsprechenden Fotographien, zumindest eingegabelt werden: Der Palügletscher stieß in den Jahren vor 1895 und zwischen 1918 und 1926 kurzfristig vor. Der Cambrenagletscher besitzt ebenfalls zwei Wallsysteme im Inneren des Gletschervorfeldes, welche zwischen 1880 und 1905 einerseits und zwischen 1908 und 1930 andererseits gebildet wurden. In der bereits 20jährigen aktuellen Vorstoßphase hat der Cambrenagletscher eine klare Stirnmoräne zusammengeschoben.

Aus Bildern und historischen Berichten geht hervor, daß die Gletscher des Berninapaßgebietes im 17., in der zweiten Hälfte des 18. Jahrhunderts und vor 1850 verhältnismäßig groß waren. Unter anderen leisten besonders die Darstellungen von H.C. ESCHER VON DER LINTH sehr gute Dienste: Vom 14. August 1793 sind drei Bilder aus dem Berninapaßgebiet erhalten, für deren Geländeaufnahmen ESCHER nebst dem Fußmarsch von St. Moritz nach Poschiavo noch Zeit fand. Nur selten sind Bilder derart detailliert und dazu noch mit dem genauen Datum versehen. Obwohl die Gletscher des Berninagebietes leicht zu erreichen sind, konnten verhältnismäßig wenige Bilder gefunden werden. Es scheint, daß der Berninapaß vor dem Bau der Straße (Mitte des 19. Jahrhunderts) und der Bahn (an- 
fangs des 20. Jahrhunderts) fast ausschließlich dem Lokalverkehr zwischen Puschlav und Oberengadin diente. Interessant sind die Zungenlängenmessungen des Morteratschgletschers, die seit 1878 durchgeführt werden. Deutlich geht aus ihnen hervor, daß die Abnahmebeträge um die Jahrhundertwende relativ klein waren: der Gletscher dürfte beinahe den Gleichgewichtszustand erreicht haben. Markant zog sich der Gletscher 1938-1967 zurück. Seither zeichnet sich wieder ein verlangsamter Rückzug ab. Deutliche Vorstöße, welche seit dem Hochstand im Jahr 1857 zu klaren Moränenwällen führten, fanden im Gegensatz zum Palü- und zum Cambrenagletscher nicht statt.

\section{Literatur}

BEELER F. (1977): Geomorphologische Untersuchungen am Spät- und Postglazial im Schweizerischen National- park und im Berninapaßgebiet (Südrätische Alpen). Ergebnisse der wiss. Untersuch. im Schweiz. Nationalpark XV/77: 131-276.

HAEBERLI W. (1979): Holocene Push-Moraines in Alpine Permafrost. Geografiska Annaler 61A (1 und 2): 43-48. HANTKE R. (1978): Eiszeitalter, Band 1. Thun.

KERSCHNER H. (1978): Untersuchungen zum Daunund Egesenstadium in Nordtirol und Graubünden (methodische Überlegungen). Geogr. Jahresber. aus Österreich XXXVI (1975/1976): 26-49.

KINZL H. (1949): Formenkundliche Beobachtungen im Vorfeld der Alpengletscher. Veröff. d. Museum Ferdinandeum (Innsbruck) 29/29: 61-82.

PATZelt G. (1977): Der zeitliche Ablauf und das Aus$\mathrm{maß}$ postglazialer Klimaschwankungen in den Alpen. In: FRENZEL B. (1977): Dendrochronologie und postglaziale Klimaschwankungen in Europa. Wiesbaden.

\section{Literaturbesprechung}

WOLFRAM Richard (Wissenschaftliche Kommission) KRETSCHMER I. (Kartogr.Leitung): Oesterreichischer Volkskundeatlas. Sechste Lieferung, Teil 1 und 2, Bildblätter + Kommentare in Ringmappe, Verlag H. Böhlaus, Wien, 1979, DM 64.--/Teil.

Als notwendige Ergänzung zu den bereits früher erschienenen Karten zur Verbreitung historischer ländlicher Gehöfts-, Flur- und Siedlungsformen ist nun der 50-seitige Kommentar 'Historische ländliche Ortsformen" von Elisabeth Tomasi erschienen. In den einzelnen Abschnitten dieses für Geographen wichtigen Teiles des Oesterreichischen Volkskundeatlasses weist die Autorin auf Entwicklungs- und Funktionszusammenhänge der traditionellen Siedlungstypen hin, indem sie Beziehungen zwischen Orts-, Flur- und Gehöftformen sowie die Einflussfaktoren auf Siedlungsart und Ortsgestaltung aufzeigt. Elisabeth Tomasi geht dabei differenziert vor, indem sie von "naturräumlichen Gegebenheiten","'ethnischen Grundlagen", Besiedlungsprozessen, "ökonomischen und sozialrechtlichen Grundlagen", Funktionen und Formen der Siedlungen spricht.

Direkten Bezug auf die beiden Atlaskarten (Blätter 92 und 93) nehmen der Bericht über die Durchführung des Kartenentwurfes und die Karteninterpretation. Die notwendige Aktualisierung der Thematik nimmt die Autorin im Abschnitt "Jüngere
Veränderungen im Siedlungsgebiet" vor. Auf acht Tafeln werden die wichtigsten Dorf- und Siedlungstypen des traditionellen ländlichen Oesterreich bildlich dargestellt.

Im-übrigen enthält die 6. Lieferung des Kommentars Texte zur historischen Landwirtschaft (Dreschen - Austreten durch Tiere'von Richard Wolfram), zum traditionellen Brauchtum des Jahreslaufes ("Burschenschaftsbrauchtum - termingebundene Unruhnächte" von Ernst Burgstaller und Richard Wolfram), zur Volksliteratur ("Sagen von den Wildleuten" von Karl Haiding) und zur religiösen Volkskunde ('Die bedeutendsten Wallfahrtsorte Oesterreichs und Südtirols" von Dietmar Assmann).

Die mitgelieferten Karten - es handelt sich um die letzten des ganzen Werkes, dessen Edition 1959 begonnen wurde - entsprechen nur teilweise den Kommentaren; das ist eine Folge der Herausgabekonzeption volkskundlicher Atlanten. In der Mitteilung des Herausgebers an die Bezieher des Oesterreichischen Volkskundeatlasses wird ein letzter unfangreicher Kommentarband noch für das Jahr 1980 versprochen, womit auch eine abschliessende Würdigung des beispielhaft gestalteten volkskundlichen Kartenwerkes möglich sein wird.

GH 3/81 Robert Kruker, Grabs 